\title{
Determining the effect of platelet-rich plasma (PRP) on improving endoscopic sinus surgery: A randomized clinical trial study $(\mathrm{RCT})$
}

\author{
Alireza Mohebbi ${ }^{1}$, Farideh Hosseinzadeh ${ }^{1}$, Saleh Mohebbi*2, Ali Dehghani ${ }^{1}$
}

Received: 11 Nov 2018

Published: 25 Dec 2019

\section{Abstract}

Background: Rhinosinusitis is an inflammatory disease of the nasal and paranasal sinus mucosa, and it becomes chronic when it lasts longer than 3 months without symptom improvement. The aim of this study was to determine the effect of PRP on improvement of endoscopic sinus surgery.

Methods: This was a randomized clinical trial (RCT) designed and implemented on 21 patients with chronic rhinosinusitis in Rasool Akram hospital in Tehran during 2016-2017. In this study, one side of the nose was randomly selected as the control and the other side as the case, and at the end of the surgery, PRP was sprayed onto the surface where the polyp was removed in the side that was intended as the case. The nasal endoscopy findings were graded according to Meltzer's criteria before and after surgery and CT findings were classified according to the Lund-Mackay scoring system. Then, all patients were followed for 6 months. The SNOT-22 questionnaire, Meltzer's criteria, and Lund-Mackay were used for data collection. Kolmogorov-Smirnov test, Wilcoxon Signed Rank test, and MannWhitney U test were used to analyze data in SPSS-22 software. P value $<0.05$ was considered significant.

Results: The mean and standard deviation of patients' age were 36.55 and 7.91, respectively. The median Lund-McKay Pre-op CT score before treatment was 10 (9.5-11), and no significant difference was found between the 2 sides of the nose (left and right). The items that showed statistically significant improvement after surgery was SNOT-22 $(\mathrm{p}<0.05)$. The mean of Meltzer scores in each side of the nose was significantly different before and after intervention $(p<0.05)$. However, the mean of Meltzer scores in the 2 sides of the nose was not significantly different before and after intervention.

Conclusion: The treatment by PRP may be effective in reducing symptoms of patients showing recurrence of CRS symptoms following endoscopic sinus surgery subjectively but not objectively.

Keywords: PRP, Endoscopic Sinus Surgery, Randomized Clinical Trial Study, Tehran

Conflicts of Interest: None declared

Funding: ENT Head and Neck Research Center, Iran University of Medical Sciences

\section{*This work has been published under CC BY-NC-SA 1.0 license.}

Copyright $\odot$ Iran University of Medical Sciences

Cite this article as: Mohebbi A, Hosseinzadeh F, Mohebbi S, Dehghani A. Determining the effect of platelet-rich plasma (PRP) on improving endoscopic sinus surgery: A randomized clinical trial study (RCT). Med J Islam Repub Iran. 2019 (25 Dec);33:150. https://doi.org/10.47176/mjiri.33.150

\section{Introduction}

Chronic rhinosinusitis (CRS) is an inflammation of the mucus that covers the nasal sinuses and the surrounding area and lasts more than 12 weeks (2-4). Clinical signs of CRS include facial fullness, nasal congestion, postnasal

Corresponding author: Dr Saleh Mohebbi, mohebbi.sa@iums.ac.ir

1. ENT and Head \& Neck Research Center, The Five Senses Institute, Iran University of Medical Sciences, Tehran, Iran

2. Skull Base Research Center, The Five Senses Institute, Iran University of Medical Sciences, Tehran, Iran discharges, rhinorrhea, hyposmia or anosmia, headache, fatigue, toothache, cough, and overpressure in the ear. In the absence of treatment, it can lead to serious complications such as periosteal cellulitis, abscess, and bowls eye $(4,5)$.

$\uparrow$ What is "already known" in this topic:

Several studies have reported the effectiveness of PRP in sinonasal surgery and improvement of healing process. Since, there has been no study to date on the effect of PRP on the nasal polyp, which is a very common disease with a very high chance of recurrence, this study was conducted to examine this theory.

\section{$\rightarrow$ What this article adds:}

The present study found a strong association between PRP and improvement of healing process in nasal polyposis. Rate of recurrence in these patients was significantly decreased. 
In the United States, the epidemiology of sinusitis is considered parallel with the epidemiology of common colds: the incidence of colds is 2-3 and 6-8/person/year in adults and children, respectively. Also, in the United States, bacterial sinus infection occurs in $0.5 \%-2 \%$ of adults and $6 \%$ $13 \%$ of children. This problem has made sinusitis one of the most common infections in patients referring to doctors. In the United States, in 2009 , about $11 \%$ of adults who referred to health centers had sinusitis. Risk factors for chronic sinusitis are as follow: allergic rhinitis; asthma; nasal congestion due to polyps; foreign bodies or tumors; immune system suppression due to such diseases as agammaglobulinemia, AIDS, and chronic granulomatous disease; structural defects such as cleft palate; defects in mucocilliary system performance; and reflux (6-9).

Generally, treatment for chronic sinusitis is based on 3 principles: controlling the growth of bacteria, reducing mucosal inflammation, and reducing the flow of fluid within the sinuses. Based on existing guidelines, the main pharmacotherapeutic approaches are glucocorticosteroids and antibiotics. However, these approaches have failed many times, especially in the following cases: adult nasal polyposis, aspirin-exacerbated respiratory disease, allergic fungal rhinosinusitis, comorbid asthma, and symptomatic patients in whom maximal medical therapy has failed. Usually, in such cases, the patient should be referred to an otolaryngologist for consideration of sinus surgery (10).

In general, the most important goals in endoscopic sinus surgery are as follow: reducing the recurrence and severity of sinus infections; improving the symptoms of sinusitis; improving the airflow through nasal passages; and enhancing the sense of smell of the patient (11). An important challenge in CRS is the recurrence of the disease and the need for re-surgery. To avoid the recurrence of sinusitis and revision surgery, the following actions have been proposed: different antibiotics; long-time low-dose antibiotics; longtime low-dose steroids; nasal steroids; and different medications such as antiukotriene, antihistamine desensitization, vaccination, and platelet-rich plasma (PRP) (12). PRP is concentrated blood plasma that has plentiful amounts of platelets and is provided by the patient. PRP works by degranulating the alpha granules in platelets containing a number of growth factors which can lead to early wound healing. These growth factors accelerate healing by increasing cellular proliferation, matrix formation, osteoid production, and collagen synthesis (13). Therefore, the aim of this study was to determine the effects of PRP on improving endoscopic sinus surgery.

\section{Methods}

This was a randomized clinical trial (RCT) study designed and implemented on 21 patients with chronic rhinosinusitis in Rasool Akram hospital in Tehran during
2016-2017. The study protocol was approved by Iranian Registry of Clinical Trials (IR.IUMS.REC.1397.687). In this study, inclusion criteria for patients were bilateral chronic rhinosinusitis with polyposis, refractory to medical management, candidate for functional endoscopic sinus surgery (FESS), and age older than 18 years. The exclusion criteria also included pt. with unilateral disease, patients with platelet function disorders, thrombocytopenia and history of other comorbidities. The random number table was used to determine the PRP was used for which of the right or left sides of the nose. If the selected number of tables was paired, PRP was used for the right side of the nose, otherwise it was used for the left side.

In this study, surgery was performed in the classic manner. One side was randomly considered as the control by the researcher; and at the end of the surgery, PRP was sprayed onto the surface where the polyp was removed in the side that was intended as the case such as maxillary sinus, sphenoidal sinus, ethmoidal sinus, and frontal recess. Also, for more durability, gel like form of PRP was used. No medication therapy was done before the surgery; however, cephalexin was described for 7 days after operation.

The steps to prepare PRP was as follow: (1) 10 milliliters of patient's blood was collected; (2) 9 milliliters of blood + 1 milliliters of acid/citrate/dextrose was used as an anticoagulant; (3) blood was centrifuged 2 times at 1500 \& 2000 rpm for 10 minutes; (4) 3 to 4 milliliters of PRP was provided as the supernatant with platelet concentration of about 5 times more than normal concentration; (5) Prepared PRP was used in the form of spray and gel.

The procedure was performed only once. Then, all patients were followed for 6 months and the results were documented and analyzed after 6 months. In the research, The SNOT-22 questionnaire was used for data collection. This questionnaire was completed before and after surgery intervention for patients. All items of this questionnaire are based on a scale of 0 to 5 , where 0 defines no problems with the given symptom and 5 defines maximal problems. The SNOT-22 questionnaire has already been used in various studies and has been validated (14-16).

Also, in this study, nasal endoscopy findings were graded according to Meltzer's criteria before and after surgery. Table 1 shows Meltzer scores which are used to classify nasal endoscopic findings. Polypoid sinonasal cavity was scored endoscopically from 0-4 according to degree of involvement (17). CT findings were classified according to the Lund-Mackay. Table 2 also shows Lund-Mackay scores which is used to classify CT findings. The collected data were entered into SPSS-22 software and analyzed using Kolmogorov-Smirnov test, Wilcoxon Signed Rank test, and Mann-Whitney U test. P value $<0.05$ was considered significant. The protocol of the study was approved by Institutional Review Board and Ethics Committee of Iran

Table 1. Meltzer scores used to classify nasal endoscopic findings

Endoscopic findings (Meltzer et al., 2006) (1)

0- No polyps

1- Small polyps in the middle meatus/edema

2- Blocked middle meatus

3- Polyps extending beyond the middle meatus, without complete obstruction

4- Massive nasal polyposis 
Table 2. Lund-Mackay scores used to classify paranasal sinuses CT scan findings (1)

\begin{tabular}{lcc}
\hline Sinus System & Left & Right \\
\hline Maxillary & $0-2$ & $0-2$ \\
Anterior ethmoid & $0-2$ & $0-2$ \\
Posterior ethmoid & $0-2$ & $0-2$ \\
Sphenoid & $0-2$ & $0-2$ \\
Frontal & $0-2$ & $0-2$ \\
Ostiomeatal complex & 0 or 2 & 0 or 2 \\
Total points for each side & $0-24$ & $0-24$ \\
Scoring for all sinus systems & & \\
$0=$ With no abnormalities & & \\
1= Partial opacification & & \\
2=Total opacification & \\
For the Ostiomeatal complex: $0=$ not occluded, 2= occluded & \\
\hline
\end{tabular}

University of Medical Sciences. Moreover, the study was performed by tenets of Declaration of Helsinki (IR.IUMS.REC.1397.687).

\section{Results}

The present study was conducted on 21 patients. The mean and standard deviation of patients' age were 36.55 and 7.91, respectively. The median Lund-McKay Pre-op CT score before treatment was 10 (9.5-11), which showed no significant difference between the 2 sides of the nose $(\mathrm{p}=0.143)$.

Chart 1 demonstrates the median and interquartile range (IQR) values of before and after SNOT- 22 items and probability values. The items that showed statistically significant improvements after surgery were as follow: the need to blow nose, nasal blockage, sneezing, runny nose, cough, PND, thick, nasal discharge, ear fullness, facial pain/pressure, decreased sense of smell, difficulty in falling sleep, wake up at night, lack of good night sleep, wake up tired, fatigue, reduced productivity, reduced concentration, frustrated, sadness, and embarrassed $(\mathrm{p}<0.05)$.

Given that the results of Kolmogorov-Smirnov test showed that data did not have normal distribution, the nonparametric tests of Wilcoxon Signed Rank test (within each sides of the right and left) and Mann-Whitney U test (between the two sides of the right and left) were used to compare the Meltzer scores before and after PRP intervention. The results of Mann-Whitney $U$ test showed that the mean of Meltzer scores in the 2 sides did not have a significant difference before and after PRP intervention ( $p=0.083, p=0.326$, respectively). The results of the Wilcoxon Signed Rank test, which were used to compare the mean of Meltzer scores before and after PRP intervention within each of the sides, showed that the mean of Meltzer scores in each of the sides was significantly different before and after PRP intervention $(\mathrm{p}<0.05)$ (Table 3). Chart 2 shows the mean of Meltzer scores before and after PRP intervention. Figure 1 shows a photo of the patient before and

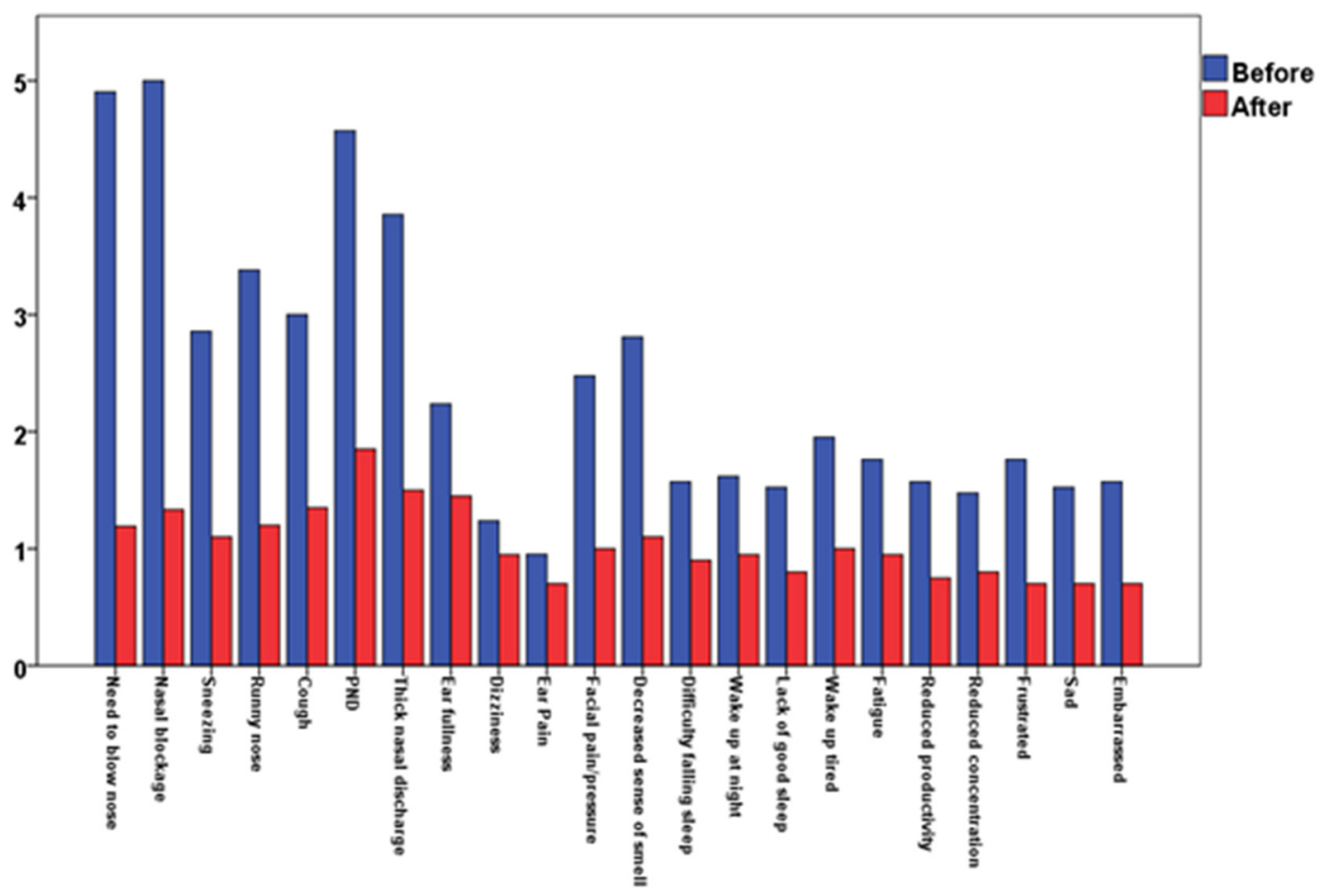

Chart 1. The Median and IQR values of SNOT- 22 items before and after the intervention 


\begin{tabular}{lccc}
\multicolumn{2}{l}{ Table 3. The Comparison the Meltzer scores before and after PRP } \\
\hline Variables & Before & After & $\begin{array}{c}\text { Wilcoxon Signed Rank test } \\
\text { P - value }\end{array}$ \\
& & & $<0.001$ \\
PRP treated side & $3(2-4)$ & $1(0-2)$ & $<0.001$ \\
Control side & $2(2-4)$ & $1(1-2)$ & \\
Mann-Whitney U test P- value & 0.083 & 0.326 & \\
\hline
\end{tabular}

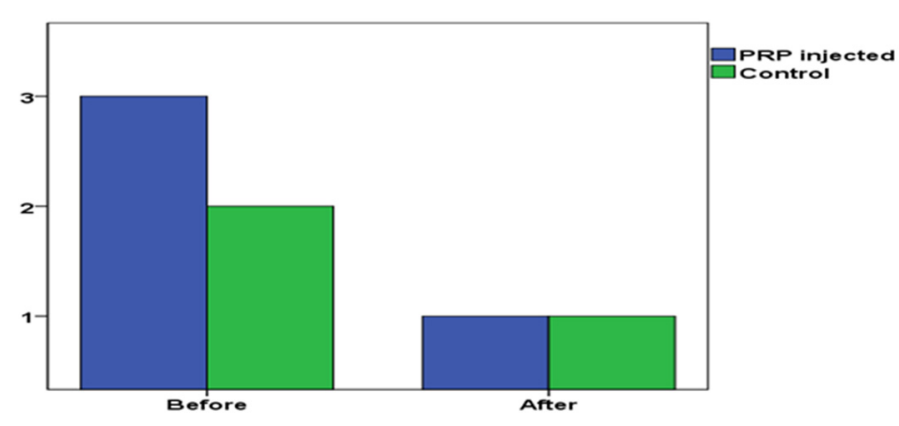

Chart 2. The Comparison the Meltzer scores before and after PRP intervention

after the surgery.
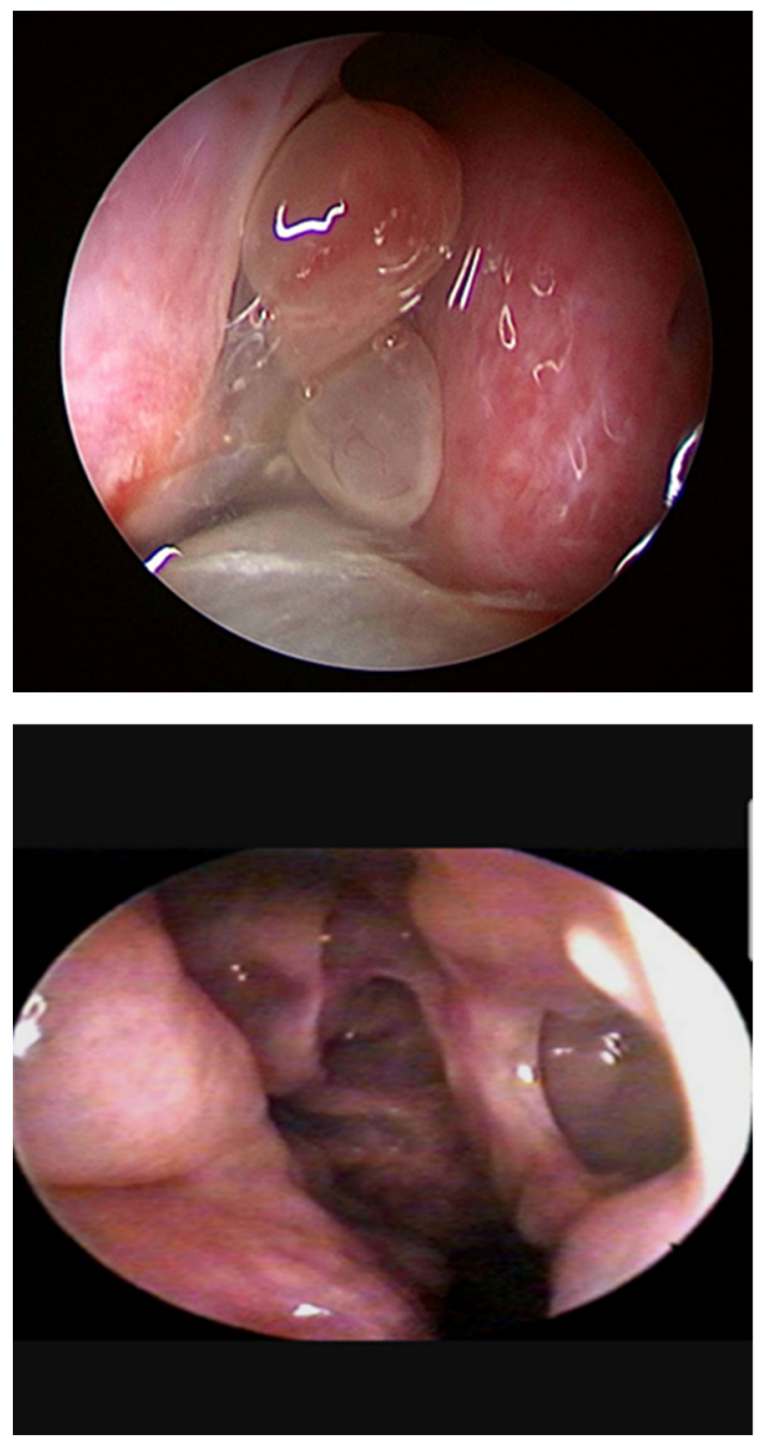

Fig. 1. A sample of the photo before and after the surgery of a patient

\section{Discussion}

Rhinosinusitis is an inflammatory disease of the sinus mucosa and nasal cavities. Chronic rhinosinusitis means that the symptoms last more than 3 months without significant improvement.

Diagnostic criteria include the presence of symptoms such as purulent nasal discharge, nasal obstruction, facial pain, pressure or fullness on frontal and maxillary sinus, hyposmia, inflammation in endoscopic examination, purulent discharge on ostium of maxillary sinus and osteomatal complex, mucosal edema of the middle meatus or ethmoidal sinus, polyps in the nasal cavity or the middle meatus, and radiographic imaging showing opacification or inflammation of the paranasal sinuses $(18,19)$. Chronic rhinosinusitis is more commonly attributed to CRS with nasal polyps (CRSwNP) and CRS without nasal polyps (CRSsNP) (20). The pathogenesis of CRS is controversial. The inflammatory process begins when bacteria, fungi, viruses, allergens, or environmental toxins interact with host environment. Defects in the normal functioning of the epithelial barriers, including the reduction of the expression of antimicrobial products, the loss of integrity of the barrier, and colonization by fungi and bacteria, have a significant effect on chronic inflammation. This chronic inflammation is characterized by increase in inflammatory cytokines and chemokine, including IL-5, thymic stromal lymphopoietin, and CCL11, which help to initiate chronic inflammatory response. Thus, gathering of immune cells, including eosinophils, mast cells, group 2 innate lymphoid cells, and lymphocytes in the chronic inflammatory response leads to nasal polyps (21-23). To date, various treatments have been developed to improve CRS. The most effective nonsurgical treatment has been intranasal steroids and nasal saline irrigation. There are inadequate data on using oral steroid in CRS without nasal polyposis. Topical antibiotics do not show any benefit in CRS without nasal polyps. Long-term antibiotics have shown possible therapeutic response after treatment for 12 weeks; however, there is concern for development of antibiotic resistant bacteria with subsequent infections. Endoscopic sinus surgery in CRS has been shown to be safe and effective when medical treatment 
has failed and in the patients with long-term maintenance medical therapy $(24,25)$. On the other hand, after FESS, patients may experience high level of recurrence of rhinosinusitis symptoms and nasal polyposis. Therefore, to improve and reduce postsurgical recurrence rate and mucosal healing, in this study, it was aimed to use PRP because of its anti-inflammatory mechanism to decrease the number of FESS revisions and decrease resistance to antibiotics.

Platelet-rich plasma consists of multiple growth and healing factors such as platelet derived growth factor, transforming growth factor, and vascular endothelial growth factor.

These agents are released by activating platelets that can be controlled by a variety of substances or stimulants such as thrombin, calcium chloride, collagen, or adenosine $5 \mathrm{c}$ diphosphat. In addition to growth factors, PRP contains fibrinogen and a number of sticky glycoproteins that support cell migration antibacterial activity by increasing the local concentration of white blood cells and antibiotic proteins, resulting in the use of products in postoperative patients as an additive to standard therapy in an effort to prevent infection.

PRP has an effective role in proliferation and differentiation of human dermal fibroblasts and myofibroblasts, which improves wound healing. Because of these properties, PRP has been used in various fields of surgery such as dental regenerative surgery, trauma, orthopedic surgery, and ophthalmology (26-28). The effects of PRP on rhinosinusitis have been studied previously. Rice et al in 2006 designed a study to assess the healing properties of platelet-rich plasma (PRP) after endoscopic sinus surgery in 30 patients with bilateral and symmetrical chronic rhinosinusitis who did not respond to medical treatment. After 13 operations, follow-up evaluations demonstrated no benefit for using PRP, and the study was terminated early. Further research was not done until Salah El Din et al in 2012 performed a randomized single blinded study on 60 patients with inferior turbinate hypertrophy. They used PRP in one group and saline in the other group (29). In their follow-up, nasal mucocilliary clearance was considered and the clearance of mucocilliary was improved in both groups, but it was statistically more significant in patients with PRP insertion. Due to positive effects of PRP and its anti-inflammatory properties, in this study, it was decided to reevaluate its effects on CRS patients after FESS intraoperatively.

According to SNOT-22, the most common symptoms which were reported by the patients were as follow: the need to blow nose, nasal obstruction, and postnasal discharges, which were reduced significantly 6 months after PRP was instilled. There was a significant difference in Meltzer score before and after treatment in the both treated and control sides. Overall analysis of patients' symptoms according to the scores recorded from SNOT-22 questionnaire data revealed statistically significant reduction after 6 months. This analysis showed the positive effect of both FESS and PRP. However, endoscopic grading of that side of the nasal cavity treated by PRP showed a difference; however, the reduction was nonsignificant when compared to the control side after 6 months.

\section{Conclusion}

Findings of this study suggested that treatment by PRP may be effective in reducing symptoms of patients showing recurrence of CRS symptoms following endoscopic sinus surgery subjectively but not objectively. Therefore, further studies should be conducted to obtain more accurate results on the effect of PRP on the nasal polyp. Also, taking more photos before and after the surgery and longer follow-ups can be helpful in studying polyp revision cases. The CSS group without a nasal polyp can also be considered to study PRP even in cases that are not candidates for surgery and can be checked for symptom relief._Moreover, it is better to have a longer follow-up. Also, it may be possible to improve PRP on the surgical site (not necessarily by spraying) and achieve the best effect of PRP on the nasal polyps. However, the above-mentioned will require further investigation in future studies.

\section{Informed consent}

Informed consent was obtained from all individual participants included in the study.

\section{Conflict of Interests}

The authors declare that they have no competing interests.

\section{References}

1. de Freitas MR, Vasconcelos DN, Freitas ÂEdHA, Maia Filho JH, e Silva CdCJBjoo. Nasal endoscopic and CT scan alterations of the paranasal sinuses as predictors of severity in patients with cystic fibrosis. Braz J Otorhinolaryngol. 2013;79(4):480-486.

2. Sedaghat AR, Phipatanakul W, Cunningham MJJjopo. Prevalence of and associations with allergic rhinitis in children with chronic rhinosinusitis. Braz J Otorhinolaryngol. 2014;78(2):343-347.

3. Wood AJ, Douglas RG. Pathogenesis and treatment of chronic rhinosinusitis. Postgrad Med J. 2010;86(1016):359-364.

4. Mustafa M, Patawari P, Iftikhar H, Shimmi S, Hussain S, Sien MJ. Acute and chronic rhinosinusitis, pathophysiology and treatment. Int J Pharm Sci Invent. 2015;4(2):30-36.

5. Hirsch AG, Stewart WF, Sundaresan AS, Young AJ, Kennedy TL, Scott Greene J, et al. Nasal and sinus symptoms and chronic rhinosinusitis in a population-based sample. Allergy. 2017;72(2):274281.

6. Blaiss MS. Pediatric allergic rhinitis: physical and mental complications. Paper presented at: Allergy Asthma Proc. 2008.

7. Tan BK, Chandra RK, Pollak J, Kato A, Conley DB, Peters AT, et al. Incidence and associated premorbid diagnoses of patients with chronic rhinosinusitis. J Allergy Clin Immunol. 2013;131(5):1350-1360.

8. Sedaghat AR, Phipatanakul W, Cunningham MJ. Prevalence of and associations with allergic rhinitis in children with chronic rhinosinusitis. Int J Pediatr Otorhinolaryngol. 2014;78(2):343-347.

9. Sih T, Mion O. Allergic rhinitis in the child and associated comorbidities. Pediatric Pediatr Allergy Immunol. 2010;21(1-Part-II).

10. Bachert C, Zhang L, Gevaert P. Current and future treatment options for adult chronic rhinosinusitis: focus on nasal polyposis. J Allergy Clin Immunol. 2015;136(6):1431-1440.

11. Al Badaai Y, Valdés C, Samaha M. Outcomes and cost benefits of functional endoscopic sinus surgery in severely asthmatic patients with chronic rhinosinusitis. J Laryngol Otol. 2014;128(6):512.

12. Nakayama T, Asaka D, Kanaya H, Kuboki A, Haruna SI. Prognostic factors for recurrence after endoscopic sinus surgery for chronic rhinosinusitis with nasal polyps. Auris Nasus Larynx. 2016;43(6):641647.

13. Rice DH. Platelet-rich plasma in endoscopic sinus surgery. Ear Nose Throat J. 2006;85(8):516.

14. Kennedy JL, Hubbard MA, Huyett P, Patrie JT, Borish L, Payne SC Sino-nasal outcome test (SNOT-22): a predictor of postsurgical 
improvement in patients with chronic sinusitis. Ann Allergy Asthma Immunol. 2013;111(4):246-251. e242.

15. Schalek P, Otruba L, Hahn A. Quality of life in patients with chronic rhinosinusitis: a validation of the Czech version of SNOT-22 questionnaire. Eur Arch Otorhinolaryngol. 2010;267(3):473-475.

16. Jalessi M, Farhadi M, Kamrava SK, Amintehran E, Asghari A, Hemami MR, et al. The reliability and validity of the persian version of sinonasal outcome test 22 (snot 22) questionnaires. Iran Red Crescent Med J. 2013;15(5):404.

17. Meltzer EO, Hamilos DL, Hadley JA, Lanza DC, Marple BF, Nicklas RA, et al. Rhinosinusitis: Developing guidance for clinical trials. Otolaryngol Head Neck Surg. 2006 Nov;135(5 Suppl):S31-80.

18. Daramola OO, Lidder AK, Ramli R, Chandra RK, Shintani-Smith S, Conley DB, et al. Patient knowledge and perception of computed tomography scan in the management of chronic rhinosinusitis symptoms. Laryngoscope. 2015;125(4):791-795.

19. Garneau J, Ramirez M, Armato SG, 3rd, Sensakovic WF, Ford MK, Poon CS, et al. Computer-assisted staging of chronic rhinosinusitis correlates with symptoms. Int Forum Allergy Rhinol. 2015;5(7):637642 .

20. Hirsch AG, Stewart WF, Sundaresan AS, Young AJ, Kennedy TL, Scott Greene J, et al. Nasal and sinus symptoms and chronic rhinosinusitis in a population-based sample. Allergy. 2017;72(2):274281.

21. van Drunen CM, Mjosberg JM, Segboer CL, Cornet ME, Fokkens WJ. Role of innate immunity in the pathogenesis of chronic rhinosinusitis: progress and new avenues. Curr Allergy Asthma Rep. 2012;12(2):120126.

22. Peric A, Gacesa D. Etiology and pathogenesis of chronic rhinosinusitis. Vojnosanit Pregl. 2008;65(9):699-702.

23. Stevens WW, Lee RJ, Schleimer RP, Cohen NA. Chronic rhinosinusitis pathogenesis. J Allergy Clin Immunol. 2015;136(6):1442-1453.

24. Rahman T, Alam MM, Ahmed S, Karim MA, Rahman M, Wahiduzzaman M. Outcome of Endoscopic Sinus Surgery in the Treatment of Chronic Rhinosinusitis. Mymensingh Med J. 2016;25(2):261-270.

25. Chen FH, Zuo KJ, Guo YB, Li ZP, Xu G, Xu R, et al. Long-term results of endoscopic sinus surgery-oriented treatment for chronic rhinosinusitis with asthma. Laryngoscope. 2014;124(1):24-28.

26. Guney A, Akar M, Karaman I, Oner M, Guney B. Clinical outcomes of platelet rich plasma (PRP) as an adjunct to microfracture surgery in osteochondral lesions of the talus. Knee Surg Sports Traumatol Arthrosc. 2015;23(8):2384-2389.

27. Gonzalez M, Arteaga-Vizcaino M, Benito M, Benito M. [Application of platelet rich plasma (PRP) and its derivatives in dental implantologie and plastic surgery]. Invest Clin. 2012;53(4):408-418.

28. Albanese A, Licata ME, Polizzi B, Campisi G. Platelet-rich plasma (PRP) in dental and oral surgery: from the wound healing to bone regeneration. Immun Ageing. 2013;10(1):23.

29. Salaheldin AH, Hussein A. Effect of platelet-rich plasma on nasal mucociliary clearance after submucous diathermy of inferior turbinate. Egypt J Ear Nose Throat Allied Sci. 2012;13(2):71-75. 